\title{
Evaluación de fertilización orgánica en cafeto (Coffea arabica) con pequeños productores de Santander, Colombia
}

\section{Evaluation of organic fertilizers in coffee (Coffea arabica), in small holdings of Santander, Colombia}

\author{
Ana T. Mosquera ${ }^{*}$, María M. Melo² , Cristian G. Quiroga ${ }^{3}$, Diego M. Avendaño³, Mónica Barahona³, Fabián \\ D. Galindo 3 , Jhonattan J. Lancheros ${ }^{3}$, Sol A. Prieto ${ }^{3}$, Andrea Rodriguez ${ }^{3}$, Danithsa N. Sosa ${ }^{3}$ \\ Recibido para publicación: Abril 20 de 2015 - Aceptado para publicación: Abril 22 de 2016
}

\begin{abstract}
RESUMEN
La agricultura en la actualidad tiene el reto de suplir la demanda existente por alimentos a nivel mundial, como también, proporcionar un desarrollo sostenible para la humanidad. Para el presente trabajo el modelo escogido fue café, generando la hipótesis que al utilizar abonos orgánicos para la fertilización del cultivo, se obtendría igual o mejores resultados que al aplicar la fertilización tradicional de la zona de estudio. Para lo cual se plantearon los siguientes objetivos: Evaluar el efecto del bocashi y lombricompuesto como fertilizantes orgánicos en el desarrollo vegetativo y sanidad del cultivo de cafeto; y determinar la influencia de la temperatura en la elaboración de bocashi. Se trabajó un diseño de Bloques Completos al Azar (BCA); los tratamientos fueron: bocashi, lombricompuesto y fertilización tradicional orgánica y las variables evaluadas fueron: altura y número de hojas por planta, así como, incidencia de patógenos fungosos. Se escogieron tres fincas ubicadas en diferentes sectores del municipio de Ocamonte-Santander para tener mayor representación de las condiciones agroclimáticas de la región, donde se estableció la variedad Castillo. Se realizó análisis físico-químico de los suelos, para determinar los nutrientes disponibles a las plantas al momento de la siembra, igualmente, se hizo análisis químico a los abonos orgánicos para determinar su influencia sobre las variables evaluadas. Los resultados obtenidos permitieron aceptar la hipótesis, y aunque químicamente el bocashi registró mayores valores que el lombricompuesto, este último, indujo mejor respuesta en el desarrollo vegetativo del cafeto.
\end{abstract}

Palabras calve: Bocashi, lombricompuesto, café orgánico, agricultura sostenible.

\begin{abstract}
Today, the agricultural sciences face the double challenge of meeting the increasing global food demand and ensuring a sustainable development for humanity. In this study we focused on the coffee production system, evaluating the hypothesis of whether, in the region of the study, the use of organic fertilizers for this crop offers equivalent or better results than those obtained by using traditional fertilizers. Specifically, we aimed to: Evaluate the use of bocashi (fermented rice bran) and worm compost as organic fertilizers to promote vegetative growth, and plant health in the coffee crop; and determine the influence of temperature in preparing bocashi. We applied a randomized complete blocks design with three treatments: bocashi, worm compost and traditional organic compost. The evaluated variables were: plant height, number of leaves per plant, and the incidence of fungal pathogens. Three farms were chosen in different localities in the Municipality of Ocamonte-Santander to cover the agro-climatic conditions of the region, the Castillo variety was used. Physical-chemical soil analysis was performed in order to determine the nutrients available to plants at the moment of planting. Chemical analysis was also performed for the organic composts in order to determine their influence on the evaluated variables. Our results allowed us to accept the hypothesis. Although, the bocashi showed greater values in terms of chemical composition compared with the worm compost, the latter induced a more favorable response in vegetative development in coffee plants.
\end{abstract}

Key words: Bocashi, worm compost, organic coffee, sustainable agriculture.

\footnotetext{
1* Ph.D. Facultad de Ingeniería, Programa de Biología, Pontificia Universidad Javeriana-Cali. Tutor del Semillero de Investigación, Grupo de Competitividad e Innovación para el Desarrollo Empresarial (CIDE). fitopatologia@hotmail.com; atmosquera@javerianacali.edu.co ${ }^{2}$ M.Sc. Planificación y Administración de Desarrollo Regional, Docente Universidad Pedagógica y Tecnológica de Colombia (UPTC), Líder del Grupo de Investigación Competitividad e Innovación para el Desarrollo Empresarial (CIDE).

${ }^{3}$ Estudiantes de Ingeniería Agronómica de la Universidad Pedagógica y Tecnológica de Colombia (UPTC), Semillero de Investigación del Grupo de Competitividad e Innovación para el Desarrollo Empresarial (CIDE).
} 


\section{INTRODUCCIÓN}

El modelo agrícola convencional se ha relacionado con mayor productividad, no obstante, también ha sido severamente cuestionado por los problemas ecológicos y ambientales que provoca. Entre los cuales se menciona: la dependencia a combustibles fósiles con baja eficiencia energética, el creciente uso de pesticidas, la degradación y pérdida de los recursos naturales y la biodiversidad, la contaminación de los alimentos y el medio ambiente, el impacto negativo en la salud de agricultores y consumidores (Lucimar et al. 2012).

Según Pizano (2001), el café para Colombia es el principal producto agrícola que ha aportado al desarrollo económico y social del país. Sin embargo, durante el proceso de beneficiado Ilega a generar hasta 3.005 toneladas de pulpa, correspondiente al $80 \%$ del total de desechos que se producen hasta obtener el producto final. Lo cual crea problemas ambientales y económicos para las regiones productoras del grano (Vásquez et al. 2009).

Lo anterior conlleva a considerar alternativas para la producción del café, al igual que para otros alimentos, con mayor posibilidad de implementar agricultura orgánica. Esta es una alternativa viable para promover el desarrollo agrícola de forma sostenible, disminuyendo el uso y dependencia de fertilizantes y plaguicidas de síntesis química (Altieri y Nicholls 2000).

Desde la antigüedad se sabe que el uso de abonos orgánicos para el manejo del suelo, permite mejorar sus características físicas, químicas, biológicas y sanitarias, con lo cual se incrementa la fertilidad del suelo y por ende la productividad de los cultivos (Boudet et al. 2015; Piedrahita y Caviedes 2012; FONAG 2010).

El abonos orgánico fermentado de mayor uso es el Bocashi (termino en japonés que significa "fermentación suave"). A diferencia de los abonos compostados, es un producto que posee materia orgánica y minerales sin transformar totalmente. Por lo cual, los agricultores lo utilizan principalmente para aumentar los microorganismos benéficos del suelo, como también mejorar características físicas y químicas, reflejándose en la sanidad y crecimiento de las plantas (Boudet et al. 2015; Ramos et al. 2014; Rodríguez et al. 2005).

Los factores que intervienen de forma directa en su elaboración son: humedad, temperatura, $\mathrm{pH}$, aireación, tamaño de las partículas de los materiales y relación carbono/nitrógeno (FAO 2011). Se considera la temperatura el factor de mayor importancia, ya que es un indicador directo de la actividad microbiana (Haug y Tortorici 1986). El proceso inicia desde el momento que se mezclan los materiales, y transcurridas 14 horas no se deben registrar temperaturas superiores a un rango entre $40 \mathrm{a}$ $50^{\circ} \mathrm{C}$, ya que está ocurriendo una fermentación alcohólica (Ramos et al. 2014; Gómez y Tovar 2008). La práctica de voltear la mezcla a diario es obligada, pues así se disminuye temperatura y humedad, además, que permite la entrada de oxígeno que beneficia poblaciones microbianas aerobias. De lo contrario, la actividad de dichos microorganismos se reduce, se aumenta la acidez, la acumulación de dióxido de carbono y otros gases orgánicos con acción biocida (Paletski and Young 1995).

Por otro lado, el lombricompuesto es el bioabono más utilizado en la agricultura orgánica. Promueve procesos naturales en el suelo a partir de la actividad y diversidad microbiana, además de facilitar el reciclaje de nutrientes, la aireación y la infiltración del agua (Boudet et al. 2015; Pedranzani et al. 2015).

Los materiales orgánicos transformados por lombrices, se caracterizan por ser fuente de nutrientes de liberación lenta, presencia de enzimas que estimulan el crecimiento vegetal, así 
como de ácidos húmicos y sustancias reguladoras del crecimiento (Pedranzani et al. 2015; Ndegwa y Thompson 2000; Brown et al. 2000).

Evaluaciones hechas con bocashi y lombricompuesto mostraron su viabilidad para la fertilización orgánica en cafeto, con mejores resultados al combinarlos con minerales naturales (Arcila y Farfán 2011; Hernández et al. 2009). Al compostar pulpa de café, Vásquez et al. (2009) lograron un producto que cumple con los parámetros exigidos por la norma técnica Colombiana (NTC 5167, 2004), para ser utilizado como abono orgánico en el cultivo. Igual ocurrió en el trabajo de Cervantes et al. (2015) al utilizar pulpa de café compostada, mejorando las propiedades microbiológicas y de estructura del suelo.

En relación a las enfermedades fungosas en cafeto, la más limitante es la roya (Hemileia vastatrix). Para lo cual, Fedecafé (2010) diseñó un plan de choque al crear la variedad "Castillo", material con resistencia genética al patógeno (Cenicafé 2011). Sin embargo, todos los materiales de cafeto cultivados en Colombia son susceptibles al hongo Cercospora coffeicola, que ataca hojas, ramas y frutos. La variedad Castillo es fuertemente afectada por este patógeno, causando lesiones circulares con borde de color ladrillo y centro claro, en algunos casos desarrolla halo clorótico (Alvarado y Monroig 2007). La germinación de conidias y su esporulación se correlacionan con valores altos de precipitación, temperatura y humedad relativa (Alvarado y Monroig 2007; Jacome y Schuh 1993).

La hipótesis planteada para el presente estudio fue, que al utilizar abonos orgánicos tipo Bocashi y Lombricompuesto para la fertilización de cafeto en sistemas de producción de Ocamonte-Santander, se tendría iguales o mejores resultados que al utilizar la fertilización tradicional de la región. Dicho planteamiento se podía observar en el desarrollo vegetativo de las plantas y sanidad del cultivo. Para lo cual se plantearon como objetivos: 1. Evaluar el efecto del Bocashi y Lombricompuesto como fertilizantes orgánicos en el desarrollo vegetativo y sanidad del cultivo de cafeto; 2. Determinar la influencia de la temperatura en la elaboración de Bocashi y los parámetros cualitativos en el Lombricompuesto, para ser evaluados como abonos orgánicos en cafeto.

\section{MATERIALES Y MÉTODOS}

\section{Localización en campo}

El estudio se realizó en el municipio de Ocamonte, departamento de Santander, a una altura de $1600 \mathrm{msnm}$, temperatura promedio de $20^{\circ} \mathrm{C}$ y precipitación pluvial promedia anual de $2.653 \mathrm{~mm}$. Se escogieron tres fincas en veredas diferentes para tener mayor representación de las condiciones agroclimáticas de la zona, las cuales fueron identificadas como: Finca uno (F1), ubicada en la vereda El Altillo con latitud $6,37^{\circ} 65^{\prime} 84^{\prime \prime} \mathrm{N}$ y longitud $73,14^{\circ} 61^{\prime} 22^{\prime \prime} \mathrm{W}$; Finca dos (F2), en la vereda Aguas frías latitud $6,36^{\circ} 92^{\prime} 21^{\prime \prime} \mathrm{N}$ y longitud $73,14^{\circ} 12^{\prime} 77^{\prime \prime} \mathrm{W}$; Finca tres (F3), en el sector las flores latitud $6,34^{\circ} 69^{\prime} 45^{\prime \prime} \mathrm{N}$ Y longitud $73,11^{\circ} 67^{\prime} 21^{\prime \prime} \mathrm{W}$.

\section{Diseño estadístico, evaluación del desarrollo vegetativo y sanidad de las plantas}

En cada finca se establecieron tres parcelas, y cada una fue sembrada con 100 plantas de cafeto de tres meses de desarrollo. Se utilizo un diseño de Bloques Completos al Azar (BCA), donde las parcelas fueron los bloques; los tratamientos los abonos orgánicos (Bocashi, Lombricompuesto y fertilización tradicional orgánica implementada por el agricultor de la región = estiércol vacuno, pulpa de café y cal dolomita); y las variables evaluadas fueron altura y número de hojas por planta, así como, incidencia de patógenos fungosos.

Las dosis al momento de la siembra para Bocashi y Lombricompuesto fueron respectivamente 300 y 250 g/planta; en la segunda y tercera aplicación 
se aumentaron a 350 y $300 \mathrm{~g} / \mathrm{planta}$; y en la cuarta a 400 y 350 g/planta. El incremento paulatino de las dosis, se hizo según recomendaciones propuestas por Arcila y Farfán (2011), que tienen en cuanta la demanda nutricional de la planta acorde a su desarrollo vegetativo.

Las variables fueron evaluadas cada dos meses antes de aplicar los abonos orgánicos, seleccionandoal azar 20 plantas por tratamiento, para un total de cuatro mediciones durante el año de estudio. Con los datos de altura y número de hojas de plantas, se realizó un análisis de varianza univariado (ANOVA) para determinar diferencias estadísticas entre tratamientos. Se aplicó la prueba de comparación múltiple de Tukey, para determinar el mejor tratamiento con el paquete estadístico SAS v. 9.2e (2013).

Después de cada aplicación de abonos orgánicos, se recorrió la parcela en forma de zigzag para determinar incidencia de patógenos fungosos (número de unidades enfermas por unidad de medida; FAO 1985). Los síntomas relacionados sólo se encontraron en hojas, se describieron y el tejido se conservó en papel periódico y bolsas plásticas para ser llevado al laboratorio de Fitopatología de la Universidad Pedagógica y Tecnológica de Colombia. Para la identificación hasta género de los hongos, se realizaron cámaras húmedas, igualmente, se sembraron en cajas de Petri con medio de papa, dextrosa y agar acidulado (PDAa) cortes de tejido vegetal sintomático. Las cajas fueron incubadas a $28{ }^{\circ} \mathrm{C}$, hasta el crecimiento de colonias y posterior observación al microscopio de estructuras de reproducción (FAO 1985). La identificación se hizo con la clave taxonómica de Barnett y Hunter (1972) y Agrios (1991).

\section{Elaboración de abonos orgánicos: Bocashi y Lombricompuesto}

Para la elaboración del Bocashi, se dispusieron bajo techo los materiales en pilas: gallinaza (100 kg), pulpa de café (100 kg), suelo de bosque (50 kg), vástago de plátano $(50 \mathrm{~kg})$ y ceniza $(50 \mathrm{~kg})$. No se excedió una altura de 50 a $80 \mathrm{~cm}$, mezclando los materiales para garantizar la homogeneidad en el proceso de descomposición. Posteriormente se aplicó agua con melaza (1 kg) y levadura (15 g) como fuente energética, para estimular la actividad de los microorganismos presentes en el material orgánico (FAO 2011). Al final se verificó el contenido de humedad, aplicando la prueba de puño (comprimir un puñado de abono para verificar presencia de humedad, sin que gotee agua; FAO 2011). La pila se cubrió con plástico negro para contribuir al aumento de la temperatura y transformación del material orgánico, como también para evitar disturbios por factores externos y pérdida de nutrientes por volatilización (Gómez 2000).

En este proceso el parámetro evaluado fue la temperatura, por ser el indicador directo de la actividad microbiana (Haug y Tortorici 1986). Los registros se tomaron a diario a la misma hora $(6: 00 \mathrm{am})$ en las tres fincas, utilizando un termómetro de laboratorio de $150{ }^{\circ} \mathrm{C}$. Para el análisis de información se tomó como referente los datos de la temperatura registrada en la Finca 1 (F1), porque durante el proceso de elaboración se mantuvieron condiciones homogéneas. Registrada en la Finca 1 (F1), porque durante el proceso de elaboración se mantuvieron condiciones homogéneas en las tres fincas.

En el caso del lombricompuesto, se siguió la metodología de Mendoza (2008), ya que propone el uso de pulpa de café como principal material orgánico y lombriz californiana (Eisenia foetida) utilizando $2 \mathrm{~kg} \mathrm{~m}^{2}$ de material. Se construyeron camas de madera para disponer los materiales orgánicos (gallinaza $(50 \mathrm{~kg}$ ), pulpa de café (100 kg) y vástago de plátano (100 kg) y evitar que las lombrices profundizaran en el suelo o fueran afectadas por factores bióticos. Finalmente se humedeció solo con agua y se cubrió con plástico negro para protegerlo de temperaturas extremas ambientales. Los 
parámetros evaluados fueron características cualitativas como: color, textura y contenido de humedad (determinado por la prueba de puño). Los datos para la Finca 2 (F2) no se muestran porque nunca fue posible culminar el proceso debido al exceso de humedad, por lo tanto el lombricompuesto aplicado a las plantas se obtuvo en la Finca 3 (F3).

\section{Análisis físico-químico de suelos y químico de abonos orgánicos}

Al inicio del estudio, en cada finca y por cada lote donde se establecieron las plantas de cafeto, se tomaron muestras compuestas de suelo (constituida por 10 submuestras). Estas muestras fueron llevadas al laboratorio de suelos de la Universidad Pedagógica y Tecnológica de Colombia, Tunja, para caracterizarlas física y químicamente mediante un análisis completo (IGAC 2007). Lo información obtenida, permitió determinar los nutrientes potencialmente disponibles para la nutrición de las plantas de cafeto.

Igualmente, del Bocashi y Lombricompuesto elaborado en las tres fincas, se tomaron muestras cada dos meses que fueron enviadas al laboratorio de Corpoica-Tibaitatá para ser analizadas. También se determinaron los nutrientes presentes, para considerarlos como abonos orgánicos que aportarían en la nutrición de las plantas de cafeto en el presente estudio.

\section{RESULTADOS Y DISCUSIÓN}

\section{Análisis físico-químico de suelos y químico de abonos orgánicos}

Según el análisis físico-químico de las muestras de suelo (Tabla 1), los nutrientes presentes se consideran suficientes para la nutrición del cultivo de café en Ocamonte-Santander. El análisis se basa en lo planteado por Guerreo (1995), ya que los elementos mayores y menores se encuentran en concentraciones óptimas. Sin embargo, hay que señalar el alto nivel de potasio en F3, que puede generar antagonismo del magnesio, reduciendo los niveles de aprovechamiento de este elemento por parte de la planta.

Para el caso de los abonos orgánicos, El Bocashi presentó mayores niveles de N, P y $\mathrm{K}$ en comparación con el lombricompuesto (Tabla 2), lo que concuerda con lo reportado por Castro et al. (2009) y Pérez et al. (2008). Los resultados pudieron deberse a la cantidad utilizadas de pulpa de café, ya que este material suministra hasta $2 \%$ para $\mathrm{N}$ y de $0,15-1,5 \%$ para $\mathrm{P}$, siendo los valores óptimos. Mientras que el porcentaje de cenizas debe oscilar de 10 $-20 \%$, y la relación $\mathrm{C} / \mathrm{N}$ debe ser $<20$.

El contenido de $\mathrm{P}$ en ambos abonos, estuvo por encima del límite inferior del valor considerado aceptable. Sin embrago, aun cuando un abono presente altos valores de nutrientes no siempre los libera con facilidad (Castro et al. 2009). En el caso de los abonos orgánicos, existe mayor posibilidad de liberar los nutrientes que están en alta concentración, porque al ser aplicados al suelo continúan procesos de descomposición que lo permite (Román 2013).

Según Castro et al. (2009) los abonos con una mayor fracción mineral generan más cenizas, lo cual se corroboró en el lombricompuesto 69,61 y $52,17 \%$ (Tabla 2), mientras que el

Tabla 1. Características físico-químicas en suelos de los sistemas de producción de cafeto. Ocamonte Santander, 2014.

\begin{tabular}{|c|c|c|c|c|c|c|c|c|c|c|c|c|}
\hline Finca & $\mathrm{pH}$ & A & Ar & $\mathbf{L}$ & $\mathbf{N}$ & $\mathbf{P}$ & $\mathbf{K}$ & Mg & Mn & $\mathrm{Cu}$ & B & Zn \\
\hline 1 & 4,9 & 31 & 45 & 32 & 76,5 & 206,1 & 149,76 & 48 & 11,3 & 1,8 & - & 2,3 \\
\hline 2 & 4,5 & 29 & 43 & 28 & 53,89 & 94,8 & 196,56 & 50,4 & 1,44 & 3,08 & - & 3,74 \\
\hline 3 & 4,7 & 51 & 19 & 30 & 243,1 & 65,5 & 599,04 & 1874,4 & 16,64 & 1,84 & 0,18 & 16,34 \\
\hline
\end{tabular}


Tabla 2. Composición química de los abonos orgánicos Bocashi y Lombricompuesto en las fincas experimentales. Ocamonte - Santander, 2014.

\begin{tabular}{|c|c|c|c|c|c|c|c|c|c|c|c|}
\hline Finca* & $\mathrm{pH}$ & $\begin{array}{c}N \\
(\%)\end{array}$ & $\begin{array}{c}\text { P2O55 } \\
(\%)\end{array}$ & $\begin{array}{c}\mathrm{K} 2 \mathrm{O} \\
(\%)\end{array}$ & $\begin{array}{c}\mathrm{C} \\
(\%)\end{array}$ & $\begin{array}{l}\mathrm{CH} \\
(\%)\end{array}$ & $\begin{array}{l}\mathrm{CO} \\
(\%\end{array}$ & $\begin{array}{c}\text { CRH } \\
(\%)\end{array}$ & $\begin{array}{c}\mathrm{CIC} \\
\mathrm{mg} / \mathrm{kg}\end{array}$ & $\begin{array}{l}\text { C.E. } \\
\text { dS/m }\end{array}$ & $\begin{array}{l}\mathrm{C} / \mathrm{N} \\
(\%)\end{array}$ \\
\hline \multicolumn{12}{|c|}{ Bocashi } \\
\hline 1 & 8,96 & 1,23 & 3,96 & 12,94 & 12,94 & 49,77 & 17,87 & 163,19 & 24 & 34,90 & 14,98 \\
\hline 2 & 8,91 & 1,06 & 1,40 & 9,14 & 9,14 & 43,28 & 6,94 & 134,93 & 12,96 & 25,60 & 6,52 \\
\hline 3 & 8,89 & 2,09 & 1,92 & 14,60 & 14,60 & 64,18 & 21,30 & 184,19 & 35,04 & 22,60 & 10,21 \\
\hline \multicolumn{12}{|c|}{ Lombricompuesto } \\
\hline 1 & 8,47 & 0,81 & 1,92 & 69,61 & 69,61 & 47,28 & 7,27 & 125,20 & 25,07 & 11,65 & 6,52 \\
\hline 3 & 8,96 & 0,61 & 1,02 & 52,17 & 52,17 & 54,82 & 10,37 & 176,43 & 37,04 & 22,60 & 16,99 \\
\hline
\end{tabular}

C: Cenizas; $\mathrm{CH}$ : Contenido de humedad; CO: Carbono orgánico oxidable; $\mathrm{CRH}$ : Capacidad de retención de humedad; CIC: Capacidad de intercambio catiónico; C.E: Conductividad eléctrica; C/N: Relación carbono/nitrógeno.

*Los datos para la Finca 2 (F2) no se muestan, porque nunca fue posible culminar el proceso por exceso de humedad.

Bocashi presentó valores dentro del rango normal. Por otro lado, la relación $\mathrm{C} / \mathrm{N}$ ha sido utilizada como un índice de velocidad de descomposición del abono y la posterior mineralización de sus nutrimentos. El valor de $\mathrm{C} / \mathrm{N}$ entre 10 y 20 se toman como aceptable para abonos orgánicos, y los dos abono evaluados presentaron niveles $\mathrm{C} / \mathrm{N}<20$. Por lo tanto, cumplen con las condiciones requeridas

\section{Efecto de los abonos orgánicos en el desarrollo vegetativo del cafeto}

Al momento del trasplante las plantas de cafeto presentaron homogeneidad en la altura, por lo tanto, estadísticamente no hubo diferencia entre tratamientos. A partir del muestreo dos (M2 = 2 mdt) y hasta el muestreo cuatro (M4 $=6 \mathrm{mdt})$, los mayores valores para ésta variable en los tres tratamientos, se observó en la Finca

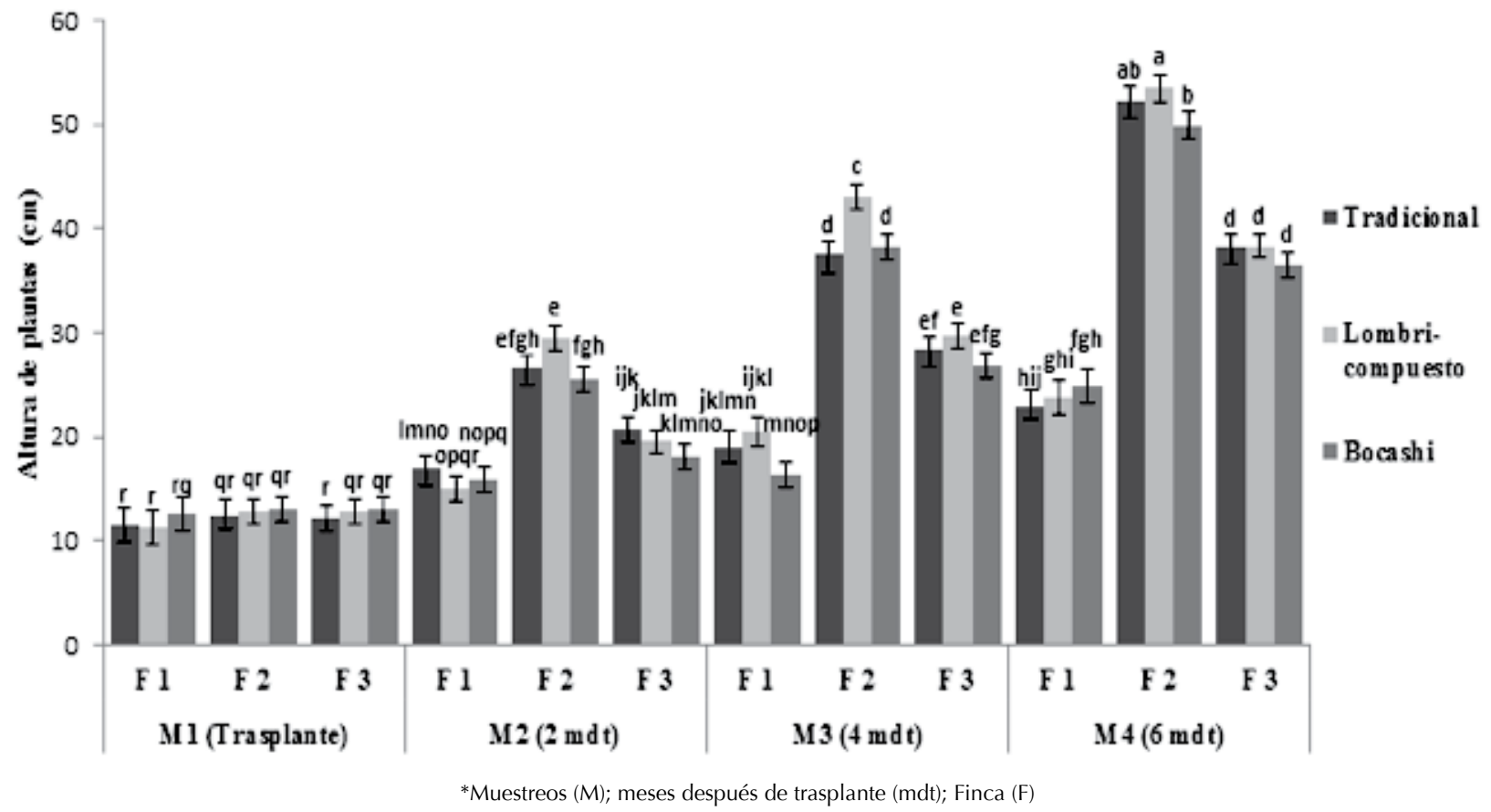

Figura 1. Altura de las plantas de cafeto durante los muestreos (M) realizados para la evaluación de los abonos orgánicos. Ocamonte-Santander, 2014. 
dos (F2). En este último periodo (M4), no hubo diferencia estadística entre el efecto del tratamiento tradicional y los abonos orgánicos preparados. Sin embargo entre estos dos, el lombricompuesto indujo mejor respuesta con valores promedios de 53,5 cm (Figura 1). Valencia (2000) afirma que, al utilizar lombricompuesto en las primeras etapas de desarrollo de las plantas de cafeto causa un aumento en su crecimiento, y dependiendo del tipo de suelo, puede resultar mejor que la fertilización química de síntesis. Además en este bio-abono existen enzimas, ácidos húmicos y sustancias reguladoras del crecimiento que estimulan el desarrollo de la planta (Pedranzani et al. 2015).

Igual como ocurrió con altura de planta, no hubo diferencia estadística entre tratamientos para el número de hojas al momento del trasplante (Figura 2). Ya en el muestreo dos (M2), las plantas de todos los tratamientos de la F2 mostraron los mayores valores; en el muestreo tres (M3), tuvieron mejor respuesta el cafeto de la F2 y la F3; en el muestreo cuatro (M4), las plantas de la F2 alcanzaron los valores más altos para esta variable. En este último muestreo (M4 = 6mdt) en la F2, aunque se registraron los valores más altos de todo el estudio con relación al número de hojas, estadísticamente no hubo diferencia entre tratamientos. Aun así, el lombricompuesto indujo mejor respuesta expresado en el valor más alto del número de hojas (42; Figura 2), seguido de la fertilización tradicional y Bocashi (38 y 37, respectivamente). Todo material orgánico que pase por el tracto digestivo de la lombriz, se transforma en un abono con alta actividad y diversidad microbiana, promoviendo procesos naturales del suelo que se reflejan en el desarrollo de las plantas (Boudet et al. 2015; Pedranzani et al. 2015. En tanto el Bocashi, por ser un abono cuyo material orgánico no está totalmente transformado, se hace lenta la liberación de los nutrientes (Boudet et al. 2015;

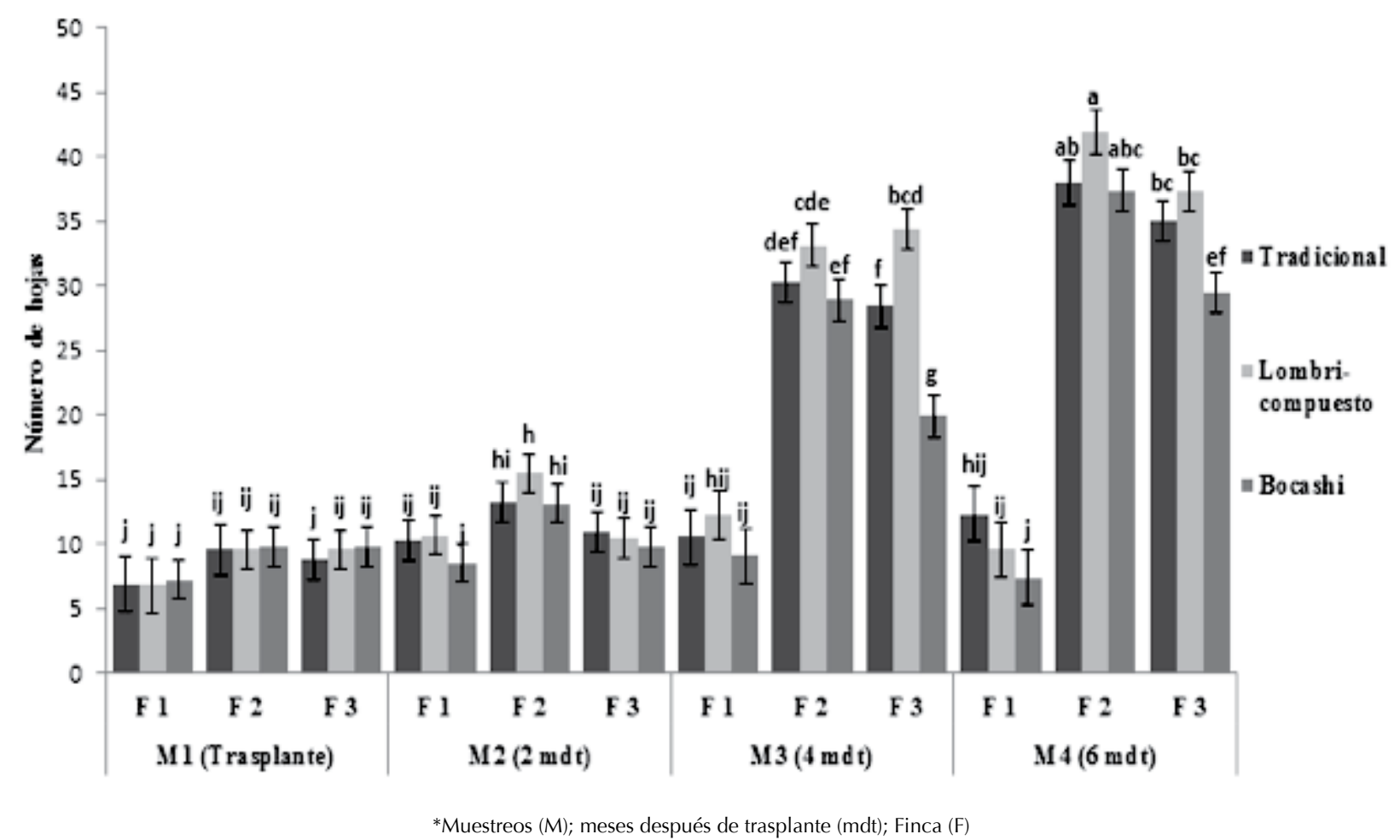

Figura 2. Número de hojas en plantas de cafeto durante los muestreos realizados para la evaluación de los abonos orgánicos. Ocamonte - Santander, 2014. 
Ramos et al. 2014b; Rodríguez et al. 2005). Además que influyen las características fiscoquímicas del suelo (Valencia 2000). Por lo tanto, al evaluar su aporte sólo con base al desarrollo de la planta, y en tan corto tiempo, no se pudo evidenciar su efecto positivo.

Se debe señalar que el análisis de composición química de los abonos orgánicos (Tabla 2), mostró al Bocashi con los mayores valores en comparación al Lombricompuesto. Sin embargo, el Lombricompuesto influyó más sobre las plantas de cafeto al registrarse los mayores valores de altura en planta y número de hojas (Figura 1 y 2 2). Lo anterior estaría indicando, que éste abono permite mayor disponibilidad de nutrientes, lo que coincide con lo mencionado por Jiménez et al. (2012). Los autores explican, que el paso del material orgánico a través del aparato digestivo de la lombriz, provocan cambios químicos de los nutrientes a formas asimilables para las plantas, principalmente el nitrógeno.

\section{Elaboración de abonos orgánicos: Bocashi y Lombricompuesto}

En la figura 3, se observa que durante los 10 primeros días en la elaboración del Bocashi, la temperatura se elevó hasta $40{ }^{\circ} \mathrm{C}$. Lo anterior se relaciona con la estabilización del proceso generando fermentación alcohólica (Ramos etal. 2014a; Gómez y Tovar 2008). Transcurridos 11 días, los registros de temperatura corresponden a la ambiental, indicando disminución de la actividad microbiológica al reducirse las fuentes energéticas para retroalimentar el proceso (FAO 2011).

Entre los 35 y 42 días aumentó nuevamente la temperatura, pero los valores no fueron superiores a $36{ }^{\circ} \mathrm{C}$, lo cual ocurre por procesos de lenta degradación de algunos materiales (FONAG 2010). Después de los 45 días, la temperatura descendió con registros constantes de $31{ }^{\circ} \mathrm{C}$. Ramos et al. (2014) indican, que los microorganismos metabolizan paulatinamente

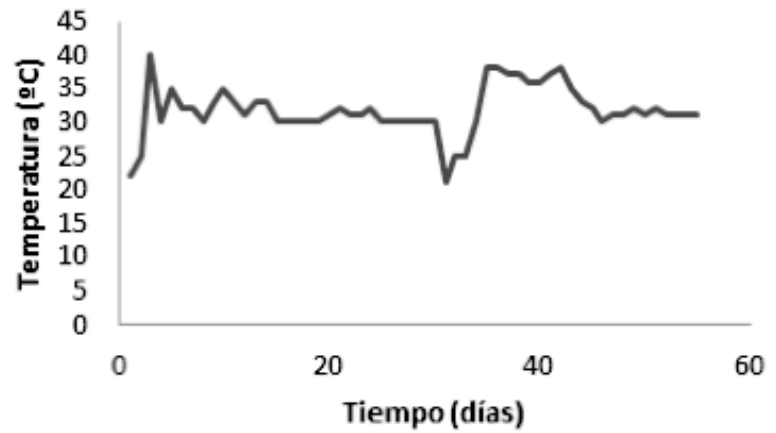

Figura 3. Registro de temperatura durante la elaboración de bocashi. Ocamonte Santander, 2014.

los sustratos carbonados, y así mismo, la temperatura desciende e inicia la etapa de maduración que puede ser prolongada.

En el bocashi no se registra etapa termofílica (temperatura entre 60 a $70{ }^{\circ} \mathrm{C}$ ), por lo cual los registros máximos de temperatura están en el rango de 40 a $50{ }^{\circ} \mathrm{C}$. Lo anterior conlleva, a que población microbiana que participan del proceso de descomposición de materiales, también cumpla con actividad antagónica contra fitopatógenos (Ramos et al. 2014; Gómez y Tovar 2008). La ecología microbiana señala la multifuncionalidad de la mayoría de los microorganismos, como estrategia de adaptación (Porras y Bayman 2011).

El lombricompuesto es el producto del excremento de las lombrices, que constituye un fertilizante orgánico suave, liviano, desmenuzado, con olor a tierra fértil húmeda y estable por periodos prolongados. Además rico en enzimas, sustancias hormonales y alto contenido de microorganismos, que lo hace superior a cualquier otro tipo de fertilizante orgánico conocido (Díaz 2002). Estas características físicas fueran observadas en el lombricompuesto elaborado para el presente estudio.

El color marrón oscuro y la prueba de puño, fueron los indicadores utilizados para considerar que el abono orgánico estaba listo cada dos 
meses, y ser utilizado como abono en cultivos de café. Giuliett et al. (2007), mencionan que un lombricompuesto está listo cuando tiene un aspecto terroso, suave, inodoro y de fácil manipulación.

El uso del plástico negro tanto en la elaboración del bocashi como del lombricompuesto, tal como lo afirma Gómez (2000), contribuyó a aumentar la temperatura para lograr la rápida degradación del material orgánico. Igualmente para evitar disturbios externos y pérdida de nutrientes por volatilización.

En este estudio los dos abonos orgánicos elaborados cumplieron con los parámetros de calidad mencionados en literatura (Ramos et al. 2014b; Giuliett et al. 2007); Díaz 2002). Además, las condiciones de temperatura y humedad del municipio de Ocamonte, junto con las prácticas de manejo implementadas, permitieron obtener en menos de dos meses (45-50 días; Figura 3) un producto estable para ser utilizado como abono en el cultivo.

\section{Sanidad del cultivo de cafeto}

En las tres fincas y en todas las parcelas donde se desarrolló el estudio, con $100 \%$ de incidencia, se observaron síntomas en hojas descritas como: lesiones pardas circulares, con borde color ladrillo y centro más claro (Figura
4A). Lo que coinciden con lo mencionado por Alvarado y Monroig (2007) como mancha de hierro. Según Guzmán y Rivillas (2005), esta enfermedad afecta plantas de cafeto en todos sus estados de desarrollo, y especialmente, a plantas que crecen en suelos con bajo contenido de nutrientes, exposición directa al sol y sin aplicación de fungicidas. Lo que concuerda con las condiciones edafoclimáticas y de manejo presentes en los sistemas de producción del estudio.

En campo, durante la descripción de síntomas se encontró daño mecánico ocasionado por una granizada ocurrida en la región (Figura 4B). Tales daños reducen el rendimiento en la planta o deprecia la calidad del producto final (FAO 2010).

En cámara húmeda sobre las lesiones de hojas, se desarrollaron conidióforos y conidias características del hongo patógeno Cercospora (Guzmán y Rivillas 2005). Los conidióforos se caracterizan por ser septados, oscuros, rectos y fasciculados (Figura 5C). Las conidias (Figura 5D) son individuales, hialinas, pluriseptadas, lisas, alargadas y tamaño variable (Agrios 1991; Barnett y Hunter 1972). La literatura reporta la especie C. coffeicola como causante de la mancha de hierro a nivel foliar (Alvarado y Monroig 2007; Guzmán y Rivillas 2005).

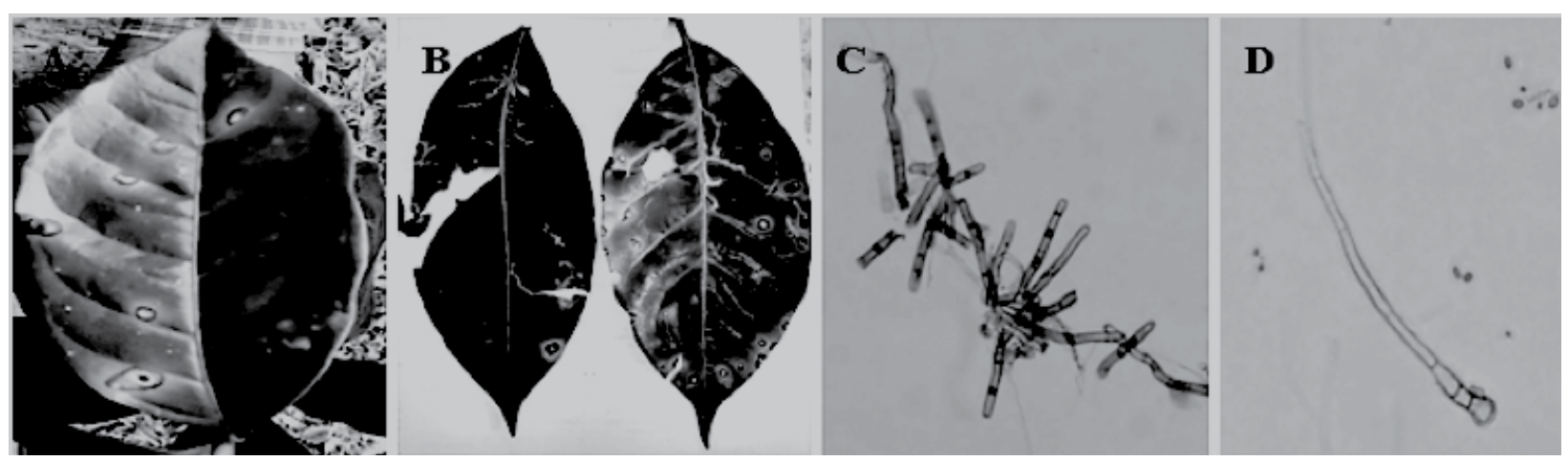

Figura 4. Hojas de cafeto afectadas por condiciones bióticas (Cercospora sp.) y abióticas. A. Síntomas de mancha de hierro. B. Daño mecánico por granizada. C. Conidióforos demateaceos agrupados. D. Conidias hialinas individuales (Foto: Barahona 2014). 


\section{CONCLUSIONES}

- Se acepta la hipótesis planteada al comprobarse estadísticamente, que el bocashi y lombricompuesto permiten obtener iguales resultados en relación al desarrollo y sanidad del cultivo de cafeto, que al utilizar la fertilización tradicional de la zona de estudio.

- Químicamente el bocashi registró mayores valores que el lombricompuesto, sin embargo, el lombricompuesto indujo mejor respuesta en el desarrollo vegetativo de las plantas corroborando lo mencionado en otros estudios.

- Las condiciones ambientales presentes en el municipio de Ocamonte y la implementación rigurosa de las prácticas de manejo, permitieron obtener bocashi y lombricompuesto a los 50 días para la fertilización orgánica del cafeto, cumpliendo con los parámetros cualitativos exigido.

- En variedad Castillo con $100 \%$ de incidencia en hojas, se observaron síntomas y signos de Cercospora spp. (C. coffeicola agente causal de mancha de hierro). Lo que confirma, que el manejo de éste fitopatógenos depende mayormente del control genético.

\section{REFERENCIAS}

Agrios, G. 1991. Fitopatología. Limusa, México, 289p.

Alvarado, A. y Monroig, M. 2007. Guía práctica de plagas y enfermedades en café. Puerto Rico. pp. 6-7.

Altieri, M. y Nicholls, C. 2000. AGROECOLOGíA. Teoría y práctica para una agricultura sustentable, 1a edición. Programa de las Naciones Unidas para el Medio Ambiente. Red de Formación
Ambiental para América Latina y el Caribe, México, 250 p.

Arcila, J. y Farfán, F. 2011. Consideraciones sobre la nutrición mineral y orgánica en la producción de la finca. Sistemas de producción de cafés en Colombia, Centro Nacional de Investigaciones en café (CENICAFÉ). pp. 201-232

Barnett, H. and Hunter, B. 1972. Illustrated Genera of Imperfect Fungi, 3a Edición. Estados Unidos. $124 \mathrm{p}$.

Boudet, A., Chinchilla, V., Boicet, T. y González, G. 2015. Efectos de diferentes dosis de abono orgánico tipo bocashi en indicadores morfológicos y productivos del cultivo de pimiento (Capsicum annuum L.) var. California Wonder. Revista Centro Agricola, 42(4): 5-9.

Brown, G., Barois, I. and Lavelle, P. 2000. Regulation of soil organic matter dynamics and microbial activity in the drilosphere and the role of interactions with other edaphic functional domains. European Journal of Soil Biology. 178p.

Castro, A., Henríquez, C. y Bertsch, F. 2009. Capacidad de suministro de N, P y K de cuatro abonos orgánicos. Agronomía Costarricense. 33: 31-43.

Cenicafé. 2011. Cultivemos café. $2 p$. http:// www.cenicafe.org/es/index.php/ cultivemos_cafe/planta/P1 [03 de Diciembre de 2014].

Cervantes, R., Ponce de León, D., Balmaseda, C., Ramón, J. y Fernández, L. 2015. Efecto de la pulpa de café Coffea arábica L., sobre suelos del macizo montañoso Guamuhaya. Revista Ciencias Técnicas Agropecuarias. 24: 38-43.

Díaz, E. 2002. Lombricultura una alternativa de producción para emprendedores 
y productores del agro. Guía de Lombricultura. Agencia de Desarrollo Económico y Comercio exterior. Municipio capital de la Rioja, Argentina, 26p.

FAO. 1985. Manual para Patólogos Vegetales. Oficina Regional de FAO para América Latina, Santiago de Chile, 278 p.

FAO. 2010. Protección contra las heladas: fundamentos, práctica y economía. http://www.fao.org/docrep/012/y7223s/ y7223s05.pdf [30 Enero 2015].

FAO. 2011. Elaboración y uso del Bocashi. Programa especial para la seguridad alimentaria (PESA) en El Salvador. http://www.pesacentroamerica.org/ biblioteca/2011/bocashi.pdf [25 Febrero 2014].

Federación Nacional de Cafeteros (Fedecafé). 2010. Plan de Choque contra la Roya. http://www.federaciondecafeteros.org/ caficultores/es/programas_ para/plan_ de_choque. [29 Noviembre 2014].

Fondo para la Protección del Agua (FONAG). 2010. Abonos Orgánicos, protegen el suelo y garantizan alimentación sana. Manual para elaborar y aplicar abonos y plaguicidas orgánicos. Agencia de los Estados Unidos para el Desarrollo Internacional (USAID), Estados Unidos, pp. 4-5.

Gómez, A. y Tovar, X., 2008. Elaboración de un abono orgánico fermentado a partir de residuos de flores (pétalos de rosa) y su caracterización para el uso en la producción de albahaca (Ocimum basilicum $\mathrm{L}$ ). Tesis, Microbiología agrícola y veterinaria y microbióloga industrial, Pontificia Universidad Javeriana-Bogotá. Colombia.

Giulietti, A., Pedranzani, H., Ruizo, M., Garbero, M. y Terenti, O. 2007. Respuesta biológica de cultivares de Pasto pongola
(Digitariaeriantha) a la enmienda en suelos con humus de lombriz. Pastos y Forrajes 30(1): 119-131.

Gómez, J. 2000. Abonos orgánicos. Impresora Feriva S.A, Colombia, 107 p.

Guerrero, R. 1995. Fertilización de clima medio. Santa fe de Bogotá. Monómeros. pp. 11-14.

Guzmán, O. y Rivillas, C. 2005. Producción In vitro de conidios de Cercospora coffeicola. Centro Nacional de Investigaciones de Café (Cenicafé). 2p.

Haug, R. and Tortorici, L. 1986. Composting process design criteria. BioCycle, United States. $27 p$.

Hernández, R., Cairo, C., Armas, J., Herrera, F., Hernández, R., Suárez, M. y Chongo, V. 2009. Efecto de abonos orgánicos y minerales naturales sobre las propiedades del suelo, el contenido de nutrimentos de la planta y el rendimiento de café oro. Revista Centro Agrícola 36(3): 21-27.

Instituto Geográfico Agustín Codazzi (IGAC). 2007. Métodos analíticos de laboratorio de suelos. Estudio general de suelos y zonificación de tierras. Departamento de Cordoba. pp. 128-144.

Jacome, L. and Schuh, W. 1993. Spore production and artificial inoculation techniques for Mycosphaerellafijiensis var. difformis. Agricultura Tropical. Trinidad y Tobago. 27p.

Jiménez, T., Agramonte, P., Ramírez, M., Pérez, M., Cárdenas, O., Pons, M. y Collado, R. 2012. Uso de humus de lombriz en la formulación de sustratos para la climatización de cultivos tropicales. Revista Centro Agrícola. 39(3): 37-44. 
Lucimar, A., Stéphane, B., Alfio, B., Guillame, O., Claire, L., Moacir, D. y Pascal, A. 2012. Relações entre agricultura orgânica e agroecologia: desafios atuais em torno dos princípios da agroecologia. Desenvolvimento E Meio Ambiente, 143.

Mendoza, L. 2008. Manual de lombricultura. México. 39p.http://www.cecytech.edu. $\mathrm{mx} / \mathrm{Pdf} /$ manuallombricultura.pdf $\quad[2 \mathrm{O}$ Agosto 2014].

Ndegwa, P. and Thompson, S. 2000. Effects of stocking density and feeding rate on vermicomposting of biosolids. Bioresource Technology. $7 p$.

Paletski, W. and Young, J. 1995. Stability measurement of biosolids compost by aerobic respirometry. Compost Science \& Utilization. 20p.

Pedranzani, H., Terenti, O., Ruiz, O., Quiroga, M. y Giulietti, L. 2015. Efecto de vermicompuestos pecuarios en algunos indicadores fisiológicos de Triticum aestivum var. buck pingo. Pastos y Forrajes. 38(4): 403-409.

Pérez, A., Céspedes, C. y Núñez, P. 2008. Caracterización física-química y biológica de enmiendas orgánicas aplicadas en la producción de cultivos en república dominicana. Revista de la ciencia del suelo y nutrición vegetal 8(3): 10-29.

Piedrahita, C. y Caviedes, D. 2013. Elaboración de un abono tipo "bocashi" a partir de desechos orgánicos sub producto de industria láctea (lacto suero). pp. 46-47.

Pizano, D. 2001. El café en la encrucijada: evolución y perspectivas. Volumen 7 . Alfaomega, Colombia, 12p.

Porras, A., Bayman, P. 2011. Hidden Fungi, Emergent Properties: Endophytes and Microbiomes. Annual Review Phytopathology. 49(1): 291-315.
Posada, C. y Osorio, N. 2003. Respuesta de plántulas de café a la fertilización foliar y la aplicación de pulpa de café compostada. Revista Facultad de Ciencias Agropecuarias. pp. 1-12.

Ramos, D., Terry, E., Soto, F. y Cabrera, J. 2014a. Bocashi: abono orgánico elaborado a partir de residuos de la producción de plátanos en Bocas del Toro, Panamá. Cultivos Tropicales. 94p.

Ramos, D. y Terry, E. 2014b. Generalidades de los abonos orgánicos: importancia del bocashi como alternativa nutricional para suelos y plantas. Cultivos Tropicales, 35(4): 52-59.

Rodríguez, M., Soto, R., Parets, E. y Alemán, R. 2005. Bocashi, una alternativa para la nutrición de la habichuela (Vigna unguiculata L. Walp sub-sp sesquipedalis L.), variedad Cantón 1 en huertos populares. Revista Centro Agricola. 32(1), 71-76.

Román, P. 2013. Manual de compostaje del agricultor; Experiencias en América Latina. Oficina Regional para América Latina y el Caribe, Santiago de Chile, pp. 17-25.

Valencia, F. 2000. Residuos de la producción cafetera para la producción y uso como abonos orgánicos. Cenicafé, Colombia, pp. 5- 14.

Vásquez de Díaz, V., López, A., Fuentes, B. y Cote, E. 2009. Aceleración del proceso de compostaje de residuos post-cosecha (pulpa) del café con la aplicación de microorganismos nativos. Revista CENIC Ciencias Biológicas. 40(4): 1-7. 